\title{
Can routine ultrasound screening performed before percutaneous dilatational tracheostomy prevent dreaded complication like bleeding in intensive care unit?
}

\section{Introduction}

Surgical Tracheostomy is one of the oldest procedure performed since 1909. It has now become the standard of care to a large extent replacing the surgical tracheostomy. The patients requiring tracheostomy in neurocritical care is around $18-20 \%$, whereas the requirement in medical intensive care is only $2-10 \% .{ }^{1}$ With this high scale of patients requiring tracheostomy, the chances of errors will be extended. The recent focus in the medical practice is to increase the safety of the patients by decreasing the fallacy, through the conduct of invasive procedures.

One of the common equipment being used for the safety during PDT is fiberoptic bronchoscopy. It helps in confirmation of midline puncture of the trachea, posterior tracheal wall injuries and other intraluminal complications. Extraluminal complications like injury to vascular structures are not monitored with bronchoscopy guided PDT. Paired anterior jugular veins and unpaired thyroid ima artery which are variable in their course and size are commonly injured and are frequent site of bleeding, leading to dreadful complication As intensivist, we should urge to improve safety and understand the vascular anatomy in a better way using ultrasound as a tool in screening the neck. Here, we present a case where on routine ultrasound screening of the anterior neck has aided in modifying plan of PDT.

\section{Case report}

A 72 year old female diagnosed of hemorrhagic stroke with capsuloganglionic bleed on computed tomography, presented with history sudden loss of consciousness and fall. Patient presented with GCS of E1V1M3, pupils were $3 \mathrm{~mm}$ and equally reacting to light. Patient was immediately intubated in the emergency room and shifted to neuro critical care unit for elective mechanical ventilation.
Volume 12 Issue 2 - 2020

\author{
Malathi Jabanathan,' Dheeraj Masapu,' \\ Deepak Rajappa,' Swaroop Gopal ${ }^{2}$ \\ 'Department of Anaesthesia, Sakra World hospital, India \\ ${ }^{2}$ Department of Neurosciences, Sakra World hospital, India
}

Correspondence: Dr DheerajMasapu, MD DM, Department of Anaesthesia, Sakra World hospital, Sy. No. 52/2 \& 52/3, Devarabeesanahalli,VarthurHobli, Bellandur, Bangalore, India, Email dheerajmasapu@gmail.com

Received: April 05, 2020| Published: April 27, 2020

In view of prolonged mechanical ventilation and tracheal toileting, she was planned for elective PDT after 48 hours. Visual examination of anterior part of the neck did not reveal any obvious abnormality or visible pulsations in front of trachea. Ultrasound screening of the neck (Figure 1) to identify vascular structures was performed, as customary practice in our hospital. A large vein of size more than 5 $\mathrm{mm}$ in diameter extending from thyroid cartilage to lower part of the neck was visualized both on transverse and longitudinal axis (Figure 2) The vein was traversing exactly in front of the trachea were the tracheostomy tube puncture as to made. As the risk of profuse bleeding was expected during the course of procedure, PDT was abandoned. The patient was taken up for surgical tracheostomy electively the next day. The tracheostomy was uneventful and weaned from ventilator in 24 hours and shifted to ward on day 5 . The patient was discharged home on day 10 .

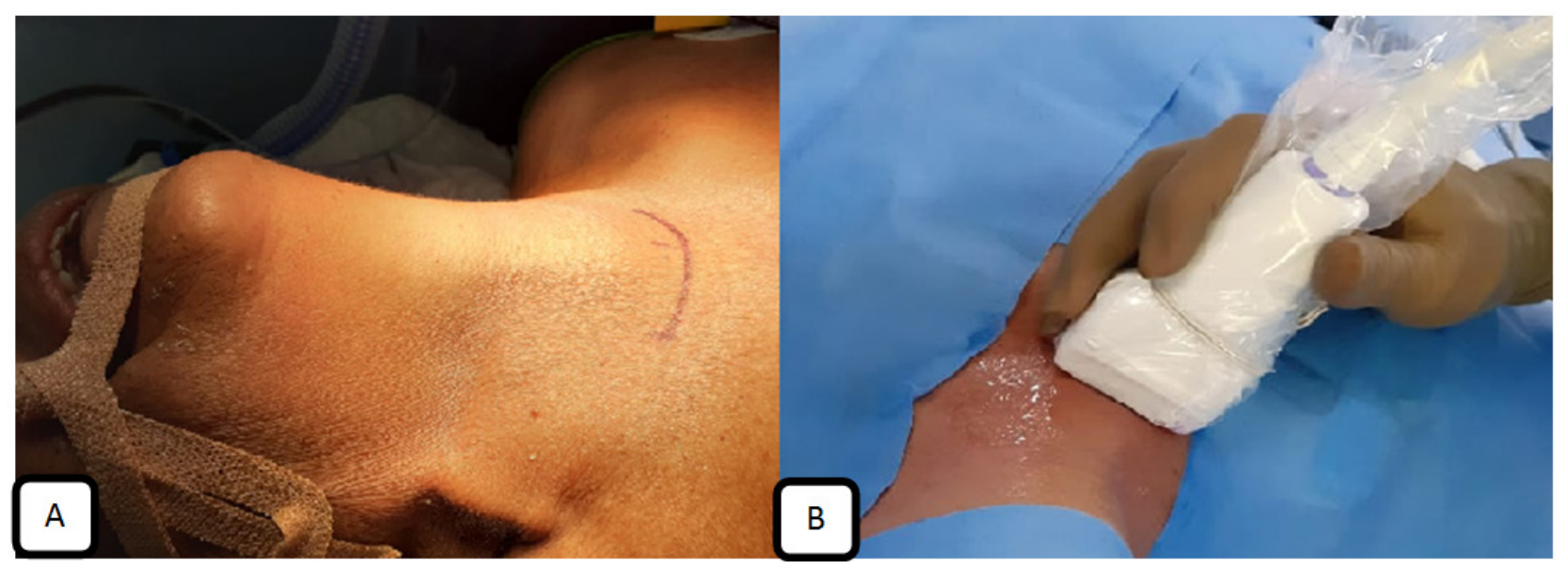

Figure I A. Patient positioned for Percutaneous tracheostomy B. Ultrasound screening of the neck. 


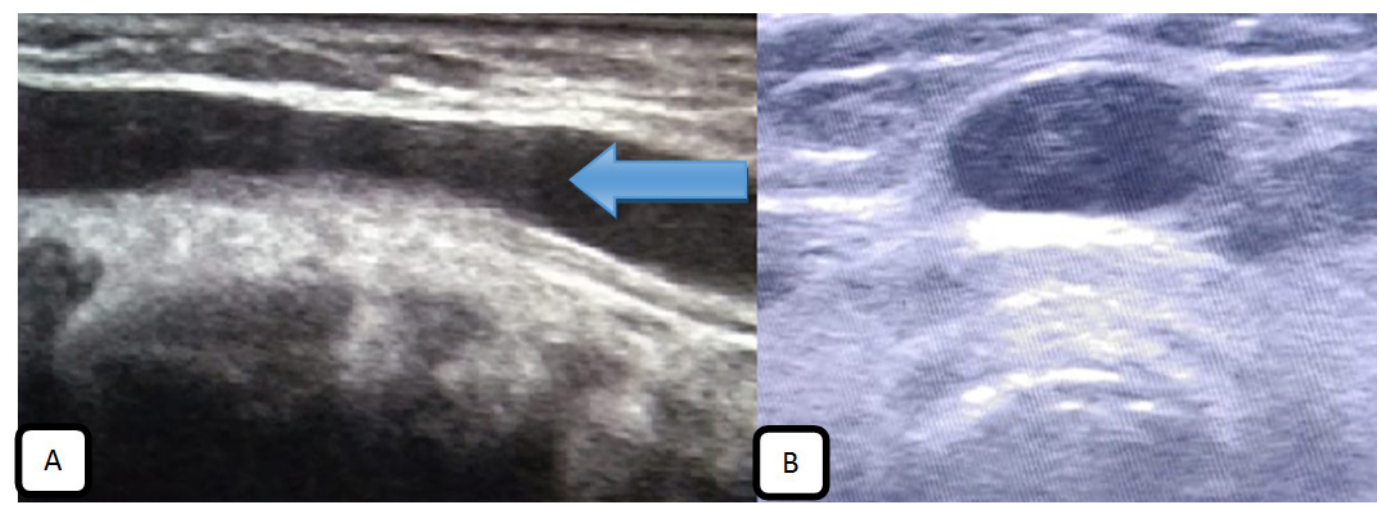

Figure 2 Ultrasound image showing anterior jugular vein A. In plane view of the vein B. Out of the plane view of the vein. The arrow shows the Anterior jugular vein.

\section{Discussion}

Even though PDT is safe and common procedure performed in ICU. A varying range of complications ranging from $11-18.2 \%$ have been shown in some studies. ${ }^{2}$ All though majority of the complications being minor complications like peristomal oozing, stomal infection. Lethal complications like bleeding, airway loss, cardiopulmonary arrest and even mortality have been reported overall compiling to a incidence of $0.17 \%{ }^{3}$ Among these lethal complications, significant bleeding constitute to $38 \%$, with $31 \%$ of these occurring during the time of insertion. ${ }^{3}$ Still intensivist are in a debate and literature in inadequate regarding ultrasound screening of vascular anatomy of neck in patients planned for PDT.

Ultrasound screening of pre-tracheal area in routine prior to PDT should entail, tracheal midline, tracheal cartilage level, thyroid isthmus and vessels anterior to trachea like anterior jugular vein, thyroid ima artery and other aberrant vessels crossing the midline (diameter and location of the vessels relative to trachea and midline) should be noted. Use of standard 7 to $10 \mathrm{MHz}$ linear probe is recommended for visualization of superficial structures of the neck. Ensure minimal compression from the probe while evaluating the vascular anatomy. The ultrasound screening done prior to a planned PDT have lead to change in the entry site of tracheal puncture in up to $50 \%{ }^{4}$

These change in the site of entry was mostly to avoid puncturing the thyroid isthmus or an aberrant vessel. We, encountered a case of large anterior jugular vein in our case, where routine ultrasound screening lead to change of plan from PDT to surgical tracheostomy in operation theatre under controlled condition. Thus preventing a major catastrophe from being happening.

\section{Conclusion}

Screening ultrasound of neck should remain a potential option for intensivists to be fully aware of any vulnerable structures to avoid complication while performing percutaneous dilatational tracheostomy. However, until prospective RCTs are conducted to evaluate the safety and efficacy of ultrasound directed PDT compared with the traditional landmark-guided technique, the best available evidence highly recommends the use of ultrasound prior to, during PDT to improve the safety of the procedure.

\section{References}

1. Toursarkissian B, Zweng TN, Kearney PA, et al. Percutaneous dilational tracheostomy: report of 141 cases. Ann Thorac Surg. 1994;57(4):862-867.

2. Hatfield A, Bodenham A. Portable ultrasonic scanning of the anterior neck before percutaneous dilatational tracheostomy. Anaesthesia. 1999;54(7):660-663.

3. Baumber R, Cohen A. Neck Ultrasound Prior to Percutaneous Tracheostomy: Should this Now be a Standard of Practice? J Intensive Care Soc. 2011;12(4):342-342.

4. Kundra P, Mishra SK, Ramesh A. Ultrasound of the airway. Indian J Anaesth. 2011;55(5):456-462. 